\title{
Blepharophimosis-intellectual disability syndrome, Ohdo type
}

INSERM

\section{Source}

INSERM. (1999). Orphanet: an online rare disease and orphan drug data base.

Blepharophimosis-intellectual disability syndrome, Ohdo type. ORPHA:2728

Ohdo blepharophimosis syndrome (OBS) is a multiple congenital malformation syndrome characterized by blepharophimosis, ptosis, dental hypoplasia, hearing impairment and intellectual disability. 